\title{
Extensión Universitaria en la Educación Syperior y su vinculación con sociedad -Estado: referentes esenciales para la transformación social
}

C Copyright 2021. Universidad Nacional Autónoma de Nicaragua, Managua (UNAN-Managua) Todos los derechos reservados

\section{University Extension in higher education and its link with society -state: essential references for social transformation}

Karla Patricia Castilla

Resp. de Extensión Universitaria UNAN-FAREM Chontales

https://orcid.org/0000-0001-7641-067X

Karla.castilla@unan.edu.ni

Concepción Mendoza Castro

Docente: Dep. Ciencias Económicas

https://orcid.org/0000-0003-3226-0715

concepción.mendoza@unan.edu.ni

Fecha de recibido: 15/04/2021
Luz Marina Solano Galeano

Docente; Ciencias de la Salud

https://orcid.org/0000-0003-3508-9394

solanoluzmarina7o@gmail.com

\section{Resumen}

En la actualidad, la extensión universitaria es reconocida como la mejor expresión de integración creativa de la universidad-sociedad y su vínculo idóneo con el estado, por lo que existe la posibilidad de hacer de esta función un eje importante de la acción de la comunidad universitaria, como el hilo conductor del compromiso social de la Universidad, por ende todos sus programas adquieren una extraordinaria relevancia en el quehacer de la UNAN Managua.

La vinculación de la universidad con la Política de extensión universitaria y sociedad consiste en el conjunto de acciones y procesos académicos ejecutados por los departamentos en cooperación con sectores externos a la universidad, como el Estado, los gobiernos locales, los sectores productivos y la sociedad civil, orientados a resolver problemas y ejecutar programas y proyectos que tengan impactos positivos en la localidad, región y país, así como en la formación profesional de los estudiantes.

Finalmente el objetivo de este artículo, es establecer el contexto del desarrollo de la extensión Universitaria de la UNAN-Managua, específicamente de la Facultad Multidisciplinaria de Chontales (FAREM-Chontales) y su vinculación con la sociedad y el estado, teniendo como premisas contribuir a la demanda de la sociedad que favorece al cumplimiento de los ejes del Programa Nacional de Desarrollo Humano 2018-2021.

\section{Palabras claves}

Extensión, vinculación, Estado, sociedad. 


\section{Summary}

Currently, the university is recognized as the best expression of creative integration of the universitysociety and its ideal link with the state; For this reason, there is the possibility of making this function an important axis of the action of the university community, as the guiding thread of the University's social commitment, therefore all its programs acquire an extraordinary relevance in the work of UNAN Managua.

The linking of the university with the University Extension Policy and society consists of the set of academic actions and processes executed by the departments in cooperation with sectors outside the university, such as the State, local governments, productive sectors and civil society. , aimed at solving problems and executing programs and projects that have positive impacts on the locality, region and, as well as on the professional training of students.

Finally, the objective of this article is to establish the context of the development of the University extension of the UNAN-Managua, specifically of the Multidisciplinary Faculty of Chontales (FAREM Chontales) and its link with society and the state, having as premises to contribute to giving response to the demand of society that favors compliance with the axes of the National Human Development Program 2018-2021.

\section{Keywords}

University extension, bonding, State, Society.

\section{Introducción}

\section{Cambia tus pensamientos y cambiarás tu mundo}

(Norman Vincent Peale)

La Extensión Universitaria en la actualidad juega un papel preponderante para el desarrollo local y del país, porque permite la vinculación de la universidad y el estado, trabajando de la mano para dar repuestas a la demanda de la población y cumplir con los objetivos de desarrollo de la agenda 2030.

En la actualidad, la extensión universitaria es reconocida como la mejor expresión de integración creativa de la universidad-sociedad y su vínculo idóneo con el Estado; por lo que existe la posibilidad de hacer de esta función un eje importante de la acción de la comunidad universitaria, como el hilo conductor del compromiso social de la Universidad, por ende todos sus programas adquieren una extraordinaria relevancia en el quehacer de la UNAN-Managua.

El trabajo en materia de extensión universitaria en la UNAN-Managua, es incuestionablemente un potencializador para dar respuestas a las demanda sociales y del estado. En este sentido la sociedad nicaragüense, está encomendando a las instituciones de educación superior, en la que se encuentra la UNAN, Managua, la resolución de problemas tanto sociales, económicos, de salud y ambientales, de forma científica y pertinente, a través de la extensión donde se involucre toda la comunidad universitaria, mediante diferentes manifestaciones tales como las mejoras de la calidad educativa, la igualdad de género, la lucha contra las enfermedades, el trabajo digno a través de la innovación y el emprendimiento.

Cabe destacar, que la UNAN-Managua, en la búsqueda del fortalecimiento de la Extensión Universitaria, ha redimensionado su concepción y su funcionamiento, para buscar una mejor sinergia entre los procesos de formación e investigación en correspondencia con las demandas sociales y los ejes del programa nacional de desarrollo humano 2018-2021.

Por consiguiente, en los Estatutos de la UNANManagua con sus Reformas, el Arto 19 establece, que:

La extensión y proyección universitaria constituyen una responsabilidad social de la institución universitaria, para extender su acción hacia las familias, la comunidad y la sociedad, para fortalecer las relaciones de cooperación e intercambio de conocimiento y creatividad de sus alumnos, docentes e investigadores por medio de las diversas acciones de innovación y emprendimiento. (UNAN, Managua, 2018, p. 24).

Finalmente, el objetivo de este artículo, es establecer el contexto del desarrollo de la extensión Universitaria de la UNAN-Managua, específicamente de la Facultad Multidisciplinaria de Chontales (FAREM Chontales) y su vinculación con la sociedad y el estado, teniendo como premisas contribuir a dar respuesta a la demanda de la sociedad que favorece al cumplimiento de los ejes del Programa Nacional de Desarrollo Humano 2018-2021. 


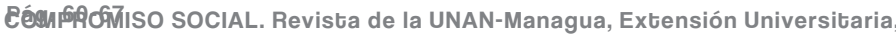

\section{Desarrollo}

\subsection{Contexto histórico. La extensión universitaria. Sus inicios}

No podemos hablar de extensión universitaria sin realizar un recorrido en la historia. Esta, tiene como antecedente el fenómeno general sociopolítico que acompaña al nacimiento del concepto teórico de extensión cultural, el cual se gesta en los últimos años del siglo XVIII y primera mitad del XIX, inspirado por el impulso, que la concepción moderna de cultura recibió tanto de los ideólogos de la Revolución Francesa como de los teóricos de la Ilustración alemana y filósofos racionalistas y empiristas ingleses (Cedeño \& Machado, 2021).

En un clima de inquietud y ebullición cultural nace el término 'extensión', acuñado por Posada en 1911, quien la concibe y define como: "Toda labor expansiva de carácter educativo y social, realizada por la Universidad fuera de su esfera oficial docente." (Cedeño \& Machado, 2021, p. 2). Este nueva redefinición venía a dar respuestas a las demandas sociales que en ese momento eran importantes y también a las inquietudes de los docentes universitarios del primer cuarto del siglo XX, quienes manifestaban su interés y preocupación por expiación la de dicha cultura.

De igual forma tiene un precedente importante en las iniciativas del profesor James Stuart de la Universidad de Cambridge a fines del siglo XIX, o de otros de la Universidad de Oxford, que organizaban clubes (Toynbee Hall) donde se intentaba que los universitarios fraternizaran con los obreros y que el ideal universitario llegara a las capas más desfavorecidas socialmente (Cedeño \& Machado, 2021).

Continuando en esa misma línea, fue hasta principios del Siglo XX los aires extensionistas también llegaron a esta área por el incremento de la presencia de europeos y norteamericanos, tanto los que llegaban con el propósito de la reconquista o los que venían a refugiarse como inmigrantes huyendo de las guerras que devastaban sus países. Ejemplos de estas influencias se encuentran en algunas universidades, como la Universidad de La Plata en la cual, desde su creación en 1905, incluían en sus estatutos la extensión con la misma jerarquía de la docencia y la investigación. Muestra también de ello fue el 1er Congreso Internacional de Estudiantes Americanos, celebrado en Montevideo en enero de 1908, en el cual abogaron por la extensión universitaria. (González \& Larrea, 2019)

\section{La extensión universitaria en Nicaragua}

El Consejo Nacional de Universidades (CNU) ha contribuido al desarrollo de la educación en Nicaragua, desde su fundación en 1990; teniendo como misión y visión la formación integral de profesionales de pre y posgrado de la más alta calidad a través del fortalecimiento del trabajo de "extensión y proyección social que vincula de manera dinámica y permanente a la comunidad universitaria con el entorno donde van a desempeñarse como profesionales, así como la búsqueda de la innovación con calidad, pertinencia e interculturalidad" (1990, p. 1)

La UNAN- Managua en conjunto con las líneas de trabajo del CNU, y en su búsqueda de elevar su calidad educativa en lo profesional formaliza la extensión universitaria con la creación de la dirección de extensión universitaria en 2011 con el objetivo de concentrar la información, coordinar los procesos y potenciar los recursos.

Las áreas que se atendía eran: UNAN-Ambiental, Innovación y Emprendimiento, creación de políticas y normativas, elaboración de convenios; es así que durante su dirección se creó la Comisión de Extensión Universitaria. (Maliaños, A, S.F)

Continuado la misma línea la UNAN-Managua, ha redimensionado su concepción y su funcionamiento, entre los procesos de formación e investigación en correspondencia con las demandas sociales y los ejes del Programa Nacional de Desarrollo Humano 20182021.

En los Estatutos de la UNAN-Managua con sus Reformas, el Arto 19 establece, que:

La extensión y proyección universitaria constituyen una responsabilidad social de la institución universitaria, para extender su acción hacia las familias, la comunidad y la sociedad, para fortalecer las relaciones de cooperación e intercambio de conocimiento y creatividad de sus alumnos, docentes e investigadores por medio de las diversas acciones de innovación y emprendimiento. (UNAN, Managua, 2018, p. 24) 


\section{La extensión Universitaria-Estado-Sociedad}

2.2.1. Vinculación con la sociedad: La vinculación de la universidad con la Política de extensión universitaria y sociedad consiste en el conjunto de acciones y procesos académicos ejecutados por los departamentos en cooperación con sectores externos a la universidad, como el Estado, los gobiernos locales, los sectores productivos y la sociedad civil, orientados a resolver problemas y ejecutar programas y proyectos que tengan impactos positivos en la localidad, región y país, así como en la formación profesional de los estudiantes. (Universidad Nacional Autónoma de Nicaragua, Managua, 2018)

EnlaXXVIII Asamblea Regional edición2019 desarrollada en Nicaragua, en relación a la extensión universitaria se plantea "El tema central nuestro gira alrededor de la Extensión Universitaria, es decir la vinculación de la universidad y sociedad, la proyección social de la Universidad, la acción social de la Universidad. Sabemos que la universidad tiene tres ejes sustantivos; Docencia, Investigación y Extensión Universitaria, todo lo que es transferencia de resultados de investigación a la sociedad es Extensión, pero también la cultura y el arte, y todo lo que la universidad ofrece en servicios ese es Extensión, entonces es ese algo que vincula a la universidad con la sociedad", expresó René Cordón, coordinador del SICAUS. (Consejo Nacional de Universidades, 2019)

Los académicos extensionistas, están bien claros que el saber está en todas partes, en la comunidad hay mucho conocimiento, y la universidad tiene que aprender mucho de ella, y a la vez investiga, produce conocimiento y tiene que transferirlo a la sociedad, pero es ese diálogo de saberes que intervienen para construir juntos proyectos de diferentes naturaleza, que permiten el cumplimiento de los objetivos de Desarrollo Sostenible, "y para lograrlos la universidad tiene un rol, pero si no trabaja en forma conjunta con la sociedad y con el Estado, entonces no se pueden alcanzar los objetivos", enfatizó Cordón.

Analizando las formas de expresión, cada país y cada universidad fueron implementando diversas acciones para lograr su relación con el medio social, asumiendo distintos roles desde el vínculo universidad-estadosociedad con un alcance territorial muy variado tanto en el ámbito local, como en el internacional, mediante diferentes metodologías, donde se abordan problemáticas o temáticas muy amplias en trabajos específicos, hasta los vinculados con el desarrollo de una comunidad, micro región, municipio e incluso aportes al progreso de un país.

El debate acerca de la extensión universitaria que se viene dando desde hace algunos años en el ámbito universitario, ha estado permanentemente asociado a las posiciones que se plantean alrededor de la esencia y pertinencia de las universidades. En la actualidad es necesario, entonces, asumir que la extensión universitaria debe sustentarse en las siguientes bases, caracterizadas por (Izaguirre Remón, 2019) de la siguiente forma:

- La extensión universitaria como función integradora y como proceso pedagógico, se expresa fundamentalmente a través de programas y proyectos, los cuales se gestionan a partir de la metodología de la promoción cultural.

- La extensión universitaria está llamada a desempeñar un papel esencial en el rescate de los saberes populares y la defensa de la identidad nacional de nuestros pueblos, en el contexto globalizador.

- Se aboga por que la extensión universitaria se asuma como un proceso desde el ser humano y para el ser humano.

- La extensión universitaria es una vía para potenciar el capital activo y el elemento socializador para el desarrollo sostenible.

- La extensión se hace factible desde el intercambio de saberes entre las comunidades intra $y$ extrauniversitarias.

La docencia y la investigación tienen en la extensión un elemento liberador y creativo del potencial humano.

- El estudiante debe ser el principal agente de cambio y por tanto protagonista de la extensión.

- La extensión universitaria debe promover que los procesos de comunicación sean interactivos e integradores, con nuevos códigos, donde se estudien, investiguen y evalúen dichos procesos.

- Todo proyecto educativo es en esencia un proyecto cultural. 
- La universidad debe contribuir a formar un ciudadano capaz de ser, conocer, hacer y relacionarse; corresponde a la extensión tributar en particular a que los individuos sean capaces de ser y convivir.

- El vínculo entre las políticas culturales y educativas en la educación superior es esencial para el desarrollo armónico de la extensión universitaria.

\section{La extensión y su relación con los compromisos de gobierno}

El Plan Nacional de Desarrollo Humano (PNDH) 2018- 2021, son estrategia y metas adquirida como compromiso de país por el Gobierno Reconciliación nacional (GRU) de Nicaragua, y para el complimiento de esas metas es importante la vinculación del estado, universidad y sociedad ya que solo unidos bajo una misma finalidad logran en desarrollo del país.

El PNDH es donde se vincula y detalla los objetivos nacionales propuestas para lograr el desarrollo humano. Existen 19 ejes que contienen los principales planes sectoriales e institucionales en los campos de educación, salud, seguridad, empleo desarrollo socioproductivo, políticas ambientales y de protección de los recursos naturales, entre otros. El Plan se enfoca en el crecimiento económico con aumento del empleo y reducción de la pobreza y las desigualdades, con estabilidad macroeconómica, con soberanía, seguridad e integración, en beneficio de las familias nicaragüenses.

La agenda 2030 para el Desarrollo Sostenible, aprobada en septiembre de 2015 por la Asamblea General de las Naciones Unidas, es un plan de acción, aprobado por la ONU, que plantea 17 grandes objetivos y 169 metas para un mundo más sostenible que favorezca el desarrollo social, la economía, el medio ambiente, así como la paz y el acceso a la justicia.

De acuerdo a la Publicación de las Naciones Unidas (2018):

Esta nueva hoja de ruta presenta una oportunidad histórica para América Latina y el Caribe, ya que incluye temas altamente prioritarios para la región, como la erradicación de la pobreza extrema, la reducción de la desigualdad en todas sus dimensiones, un crecimiento económico inclusivo con trabajo decente para todos, ciudades sostenibles y cambio climático, entre otros.
Cabe destacar que para su implementación se requiere de la participación de todos los sectores de la sociedad y del Estado (Briseño Moraga, Castilla, Jirón Guzmán, \& Espino Bravo, 2020).

Basado a todos los compromisos adquiridos por el GRU, la Universidad Nacional Autónoma de Nicaragua, Managua, ha venido fortaleciendo la extensión Universitaria con su vínculo con la sociedad y el estado, a través de diferentes acciones que vienen a dar respuestas a las necesidades del país, a continuación desglosaremos las actividades realizada desde la extensión universitaria, realizada des facultad multidisciplinaria de Chontales (FAREM), acciones que han venido contribuyendo a disminuir la pobreza, hambre cero la educación, la salud y Bienestar, Educación con calidad, Igualdad de Género, Agua limpia y saneamiento, Trabajo y crecimiento económico, reducción de las desigualdades, acciones por el clima, alianza para lograr los objetivos.

\section{- La extensión: FAREM-Chontales}

Cumpliendo con el principio de la Extensión y del precepto constitucional que la Educación es un derecho del pueblo, la UNAN-FAREM Chontales, está incidiendo en las problemáticas sociales y al cumplimiento de PNDH y ODS, a través de la extensión universitaria.

\section{- Desarrollo social}

En el entendido de que el desarrollo social es un proceso que, en el transcurso del tiempo, conduce al mejoramiento de las condiciones de vida de la población en diferentes ámbitos como salud, educación, alimentación, vivienda, vulnerabilidad, empleo, salarios, etc.

\section{- Fin a la Pobreza}

En esta acción la FAREM-Chontales ha promocionado los emprendimientos de los estudiantes: se han realizado investigaciones para mejorar la producción de las áreas rurales, se ha participado en el acompañamiento a los productores para favorecer a la tecnificación de sus fincas y dar un valor agregado a su producción.

\subsubsection{Desarrollo socio productivo}

La facultad es miembro del comité de desarrollo productivo, trabaja de la mano con el MEFCCA, donde 
los estudiantes han sido protagonistas en diferentes ferias para ofertar sus productos que desarrollan en los laboratorios de la facultad, de igual manera realizan prácticas donde brindan capacitaciones a los productores y las Mipymes a través de prácticas de las carreras de Agronomía, Agroindustrial y Ciencias Económicas que a través de las prácticas entregan a las Mipymes, manuales para el desarrollo de controles administrativos y resultados de investigaciones e Investigación Aplicada y Seminario de Graduación, además se desarrollan actividades de participación y colaboración en las Comisiones de Economía Creativa y Geoparque, Minjuve, INTUR y otras instituciones. De igual manera se participa en campañas de reforestación y agroturismo, entrega de juguetes a niñas/os de barrios y escuelas alrededor de las instalaciones de la Facultad y la Reserva Privada Estación Biológica Francisco Guzmán Pasos.

\section{- Hambre Cero}

Mediante alianza con universidades extranjera e instituciones del estado, se han realizado acciones como la mejora de la merienda escolares en diferentes escuelas primarias con el objetivo de mejorar el estado nutricional de los infantes, el promedio de población beneficiada directamente son 1950 entre niños y niñas, la preparación de los alimentos son realizado por estudiante de agroindustria, de igual forma se han promocionado y realizado huertos escolares con la integración de estudiantes de las carrera de agronomía y población beneficiada.

\section{- La salud y Bienestar}

La extensión universitaria como anteriormente se expuso tiene un papel fundamental en formar valores en sus participantes, porque la FAREM Chontales, desde la docencia articula la investigación y la extensión en el quehacer del diciente, a través de las prácticas en unidades de salud, acciones preventivas ante enfermedades vectoriales, en centros de salud, el Ilenado de fichas MOSAF, campañas de salud en escuelas y comunidades, en diagnósticos de salud, talleres en casa maternas y al personal de salud, en campañas de donación de sangre y en investigaciones de parasitosis en niños de escuelas del territorio y comarcas Cultura y tradición: en cuanto a la cultura y tradición la Facultad cuenta con un equipo de música de Troba y un grupo de danza, ha intervenido en actos culturales en escuelas, y creado un grupo de aeróbicos para la comunidad universitaria y la población en general, el grupo de música ha integrado a niños de la comunidad para impartirles clase de música y se organizó grupo de danza con niñas.

\section{- Deporte}

Se cuenta con diferentes disciplinas deportivas, de las cuales imparten clase a niños de los diferentes sectores, así mismo se brindan clases para instituciones del estado en defensa personal, esto en alianza con sociedad y el estado.

\section{- Educación en todas sus formas}

Para incidir en el cumplimiento de este eje la UNAN, Managua en coordinación con la FAREM Chontales y en alianza con las alcaldías del territorio, llevan la educación superior a los territorios con los programas Universidad en el Campo (UNICAM) y SINACAN, que son convenios que se firman con las alcaldías o instituciones del Estado para servir carreras de Enfermería, Topografía, Técnico Superior en Agronomía y últimamente los cursos de profesionalización a docentes del Ministerio de Educación o de otras instituciones del Estado como la Corte Suprema de Justicia, técnico Superior en Administración de Empresas, en Desarrollo Rural Sostenible y Topografía.

Se ha contribuido mediante la articulación de la investigación y extensión a mejorar la sostenibilidad y preservación del medioambiente y la mejora de la seguridad alimentaria y nutricional de zonas rurales.

\section{- Igualdad de Género}

La facultad ha creado la comisión de género con el objetivo de promover dentro de las acciones universitarias como un eje transversal la igualdad y prevenir cualquier tipo de violencia, se han realizado capacitaciones y docente han participado en capacitaciones sobre la temática de igualdad de género.

\section{- Propiedad y ordenamiento territorial}

La facultad ha participado en la construcción de diagnóstico para el ordenamiento de la ciudad de Juigalpa, en las cuales participaron diferentes carreras por que permitió la integración de la universidad con el estado y fortalecer ese vínculo para las prácticas de los estudiantes. 


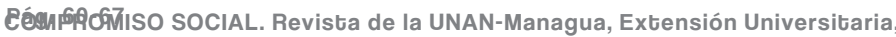

\section{- Otras acciones}

Se cuenta con programa de becas de estudiantes universitarios.

El Programa de Voluntariado y Servicio Social Comunitario a través de UNEN. Los programas de "Universidad en el Campo", "Universidad y Empresa", "UNAN-Ambiental", "Universidad Saludable" y "Seguridad Alimentaria y Nutricional". En este último, la universidad apadrina a dos escuelas donde le entregan mejora de la merienda escolar y fomenta los huertos escolares, involucrando a los estudiantes de las carreras afines, la familia, maestros y comunidad en alianza con universidad extranjera, MINED e INTA.

Ferias de salud en los distintos sectores con el objetivo de promover estilos de vidas saludables mediante: charlas para la promoción y prevención de salud, transmisible y no transmisible. Se han realizado intercambios de conocimientos con otras universidades como la BICU y URACAN, en destrezas básicas de enfermería y en proceso de atención de enfermería, administración turística y hotelera. La Facultad ha impulsado la formación de sus docentes de posgrado con doctorado y maestrías lo que ha venido a fortalecer la calidad educativa.

Todo lo anterior ha permitido situar a la Universidad como Institución de Educación Superior comprometida en el mejoramiento de la pertinencia, eficiencia, eficacia y calidad para responder a las necesidades locales, regionales y nacionales, mediante la formación integral de profesionales con competencias para construir una sociedad solidaria a favor del desarrollo humano. Cumpliendo con la política de Extensión Universitaria establece Lineamientos y Acciones Generales de conformidad con las Áreas de la Extensión Universitaria que permitirán delimitar las tareas a desarrollar desde las distintas Facultades.

\section{- Alianza para lograr los objetivos}

Para contribuir al cumplimiento de los ODS, la extensión universitaria realiza alianza desde el currículo educativo en busca de convenios o cartas de entendimiento para la realización de Prácticas de Familiarización, Especialización y Profesionalización en que estudiantes aprenden y enseñan en la praxis.

\section{Conclusiones}

Todas las acciones realizadas como parte de la extensión universitaria de la FAREM Chontales, han contribuido al cumplimiento de los compromisos del Gobierno de Reconciliación y Unidad Nacional, al dar cumplimiento a la misión y visión de la UNAN y al objetivo de ser, la extensión universitaria.

1. Han favorecido a la vinculación universidad, sociedadestado, con las prácticas profesionales los estudiantes.

2. Se han fortalecido los conocimientos de los estudiantes, aplicando lo aprendido en la teoría a la práctica.

3. Las acciones de la facultad dan respuesta a los ODS: Han mejorado el estado de pobreza de la población con acciones de mejora de la producción.

3.1 Con la mejora de la merienda y huertos escolares han disminuido el estado de desnutrición infantil y deserción escolar.

3.2 En cuando a salud las acciones han mejorado estado de salud del Binomio madre e hijo, con las campañas de donación de sangre se han evitado la muerte en la población.

4. La extensión universitaria para que tenga éxito tiene que tener aliados, y estos juntos sobre un solo objetivo y contribuir al cumplimiento de los ODS. LOS aliados de la FAREM, son Alcaldías de Chontales. INTA; INAA, MAGFROR, MINSA, MINED, POLICIA NACIONAL, EJERCITO DE NICARAGUA, MARENA, MEFCCA, PEQUEÑAS MIPYEMES, ENTRE OTRAS.

\section{Referencias bibliográficas}

- Briseño Moraga, R. A., Castilla, K. P., Jirón Gúzman, Y. M., \& Espino Bravo, M. (12 de diciembre de 2020). Programas que desarrolla la UNAN - FAREM Chontales en cumplimiento de los objetivos del desarrollo sostenible. REICe, 8(16), 102. doi:DOI 10.5377/reice.v8i16.10662

- CE. Compromiso Empresarial: La líder en innovación social. (S, F). compromiso empresarial., Electrónica. Recuperado el 21 de 02 de 2021, de compromisoempresarial.https://www. compromisoempresarial.com/rsc/2018/04/comoenfrentan-las- empresas-latinoamericanas-los-ods/ 
- Cedeño Ferrìn, J., \& Machado Ramíres, E. F. (2021). Papel de la Extensión Universitaria en la transformación local y el desarrollo social. Scielo, 12(3), 6. doi:ersión On-line ISSN 1727-8120

- Concejo Nacional de UNIVERSIDADES. (1990). CNU, Electrónica. Recuperado el 2021 de 02 de 2021, de CNU: http://www.cnu.edu.ni/historia-del-cnu/

- Consejo Nacional de Universidades. (21 de 05 de 2019). www.cnu.edu.ni. Recuperado el 18 de 02 de 2021, de http://www.cnu.edu.ni/compartiran-sobrecomo-estan-con-la- vinculación-de-la-universidad-yla-sociedad-en-cal

- Larrea, D. G. (2019). La Responsabilidad social de la universidad: antecedentes, conceptos y retos de la Extensión Universitaria. COMPROMISO SOCIAL. Revista de la UNAN- Managua, Extensión Universitaria, Pág. 13-22.

- Maliaños, A, R. M. (S.F). Unan. Recuperado el 21 de 02 de 2021, de unan: https://www.unan.edu.ni/index. php/deu/unan-managua-un-modelo-de-universidadcomprometida-socialmente.odp

- Observatorio Regional de Planificación para el Desarrollo Sostenible. (S. F). Observatorio, Electrónica. Recuperado el 21 de 02 de 2021, de Observatorio: https://observatorioplanificacion. cepal.org/es/planes/ejes-del-programa-nacional-dedesarrollo-humano-2018-2021-de-nicaragua 